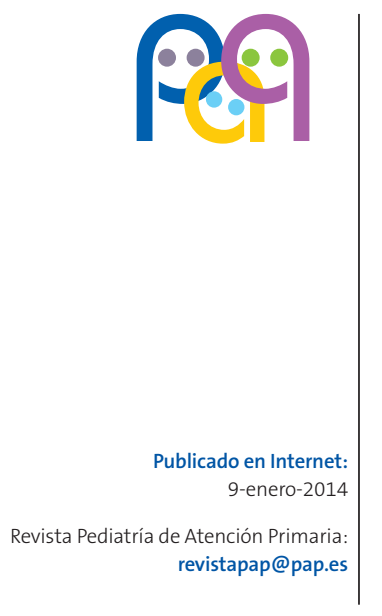

Nutrición y alimentación son dos términos que suelen usarse indistintamente, como si fueran sinónimos, de forma errónea. Nutrición hace referencia a los productos (nutrientes) que componen los alimentos e incluye un conjunto de fenómenos involuntarios que suceden tras la ingesta de los alimentos (digestión, absorción, etc.) hasta su final asimilación en las células del organismo. La alimentación, sin embargo, comprende un conjunto de actos voluntarios y conscientes que van dirigidos a la elección, la preparación e ingestión de los productos alimenticios, fenómenos complejos con un componente social extraordinariamente importante, evidente ya en los lactantes desde las primeras semanas de vida. Aquí trataremos algunos aspectos de la alimentación en este sentido.

El concepto de nutrición adecuada entendida como suficiente ha dejado de ser el objetivo en las sociedades desarrolladas, y ha sido sustituido por el de nutrición positiva u óptima, cuyos objetivos son la prevención de enfermedades crónicas y la promoción de la salud, la calidad de vida y el bienestar ${ }^{1}$.

La comunicación en el terreno de la salud se desarrolla en tantos ámbitos como los que las propias relaciones humanas dan lugar, y va mucho más
Editorial

\section{La alimentación infantil, el marketing y los medios de comunicación}

Equipo Editorial allá de las simples interacciones que se producen en el medio sanitario o en el entorno asistencial directo (en la consulta) y de la relación entre el profesional y el paciente 2 . En un mundo global como el que nos toca vivir, la conciencia de que nuestra participación es como un grano de arena en un gran desierto, debe convivir con la de que un grano tras otro puede cambiar el paisaje y al paisanaje. Siendo ambas grandes verdades, por delante tenemos un reto extraordinario nada ajeno al profesional sanitario. Las cuestiones mencionadas, sin duda de gran complejidad, difícilmente pueden ser desarrolladas en este breve texto, en el que apenas enunciaremos algunos aspectos parciales.

Pocas dudas hay acerca de la poderosa influencia de los medios de comunicación en las opiniones y actitudes de la población ${ }^{2}$. Algo parecido puede decirse de la publicidad y el marketing o mercadotecnia. La relación entre medios de comunicación y mercadotecnia es estrecha (intensa y extensa) y de necesidad mutua, así que, por conveniencias prácticas, nos referiremos a ambos fenómenos de forma conjunta ("mezclados pero no revueltos").

Una particularidad de los medios de comunicación en España es su concentración en unos pocos gru-

Cómo citar este artículo: Equipo Editorial de la Revista Pediatría de Atención Primaria. La alimentación infantil, el marketing y los medios de comunicación. Rev Pediatr Aten Primaria. 2013;15:299-303. 
pos de gestión ${ }^{3}$. Por una parte, las empresas públicas de radio y televisión, de ámbito nacional, autonómico o local, y por la otra, unos pocos grandes grupos empresariales privados ${ }^{2}$. De esto se deduce, en opinión de algunos estudiosos de la materia, una marcada tendencia a la uniformidad (y falta de pluralidad) en las informaciones, carencia de independencia (en el $49 \%$ de las informaciones, las fuentes son políticos o técnicos-políticos ${ }^{2}$ ), espacios específicos insuficientes y limitada formación especializada de los informadores ${ }^{2}$. Los estudios existentes sobre la relación entre medios de comunicación y salud se han hecho sobre los medios de comunicación impresos, y casi nada sobre otros como radio, televisión e Internet, siendo que, muy probablemente, al menos estos dos últimos, son extraordinariamente poderosos.

Esta situación condiciona, además, un tratamiento superficial, con pocas pero notables excepciones, de los temas de salud y ciencia, y una marcada tendencia a la preeminencia de temas espectaculares en detrimento de temas importantes. La publicación de suplementos o páginas especiales no ayuda, ya que no suelen despertar el interés del público, en general.

La influencia de los medios de comunicación se concreta, por ejemplo, en la sintonía que existe entre la agenda mediática (conjunto de hechos a los que se da cobertura en un contexto y tiempo determinado) y la agenda social (conjunto de prioridades o preocupaciones percibidas por la población) ${ }^{2}$.

Así, ya tenemos dibujado, al menos en sus líneas básicas, el proceso nutrición-alimentación-comunicación-publicidad-consumo (círculo ¿vicioso?).

Volvamos a la alimentación. La dieta y la actividad física son probablemente los principales determinantes del estado de salud de las personas en nuestro tiempo. Juegan un papel relevante en el desarrollo de la obesidad, el cáncer y las enfermedades cardiovasculares, es decir, de las causas principales de morbilidad y mortalidad, además del gasto sanitario y social ${ }^{1}$.

A nadie se le escapa que los cambios en la epidemiología de estas enfermedades en las últimas décadas (el incremento espectacular de su prevalencia), no pueden ser explicados por otras causas sino que bien pueden ser atribuidas a los cambios en el entorno físico, social y cultural en el que nos movemos ${ }^{1}$. Nuestros hábitos de alimentación y modos de vida han cambiado y están en la base de los cambios epidemiológicos citados antes.

Resulta chocante algo que muestran los estudios de percepción social, y que es la paradoja de que para los ciudadanos la salud es una prioridad pero a la vez se sienten poco o mal informados ${ }^{2}$. Y aquí, precisamente, radica la vulnerabilidad de los individuos ante las "fuerzas del bien y del mal", es decir, ante las influencias que ejercen los agentes sociales, entre los que se encuentran los sectores industriales de alimentación y bebidas, los medios de comunicación, las instancias políticas y, en último lugar y a distancia, las científicas.

En los medios de comunicación generalistas, la presencia de las informaciones de ciencia y salud ha crecido en cantidad, pero no en calidad ${ }^{4}$, aunque con dignas excepciones -justo es mencionarlas, y también señalar que se suelen localizar en medios de alcance local o regional-. El tratamiento en los grandes medios o espacios informativos, a los profesionales les parece en general "trivial y sobresimplificada" " hoy día el tipo de información sobre salud y el modo de hacerla "parece condenar a los lectores a la pobreza informativa, a la falta de pluralidad, de equilibrio y de profundidad" $[\text { sic }]^{5}$ y a crear expectativas infundadas ${ }^{4}$. En palabras de un experto (J. Basulto): "Los titulares relacionados con la nutrición no siempre son fiables. En ocasiones trivializan sobre asuntos importantes y otras veces alertan en exceso" $[\mathrm{sic}]^{6}$. Titular erróneo ${ }^{7}$, interpretación errónea de un estudio científico ${ }^{8}$, y presentación mediante titulares que pueden llevar a la catástrofe de, por ejemplo, afirmar que "un vaso de vino al día reduce el riesgo de tener una depresión" 9 .

Un estudio llevado a cabo en el Reino Unido y publicado en 2012 evaluó la calidad de los consejos dietéticos que aparecieron en una semana escogida al azar en los diez periódicos más vendidos del país (tanto periódicos de gran formato como The 
Times, como diarios sensacionalistas como Mirror). Se tomaron como referencia las recomendaciones de instituciones científicas reconocidas (World Cancer Research Fund [WCRF] y Scottish Intercollegiate Guidelines Network [SIGN]) y encontraron que la mayoría (68-72\% de 111) de los consejos dietéticos evaluados no tenían sustento científico suficiente; es de destacar, aunque se esperaba, que la calidad era mayor en los periódicos de gran formato que en los diarios sensacionalistas ${ }^{10}$.

Resulta curioso comprobar cómo los avances genéticos atraen la atención de los medios de comunicación y llenan espacios informativos, al parecer con gran respuesta de la audiencia. Presentan los estudios y a los investigadores como héroes o guerreros involucrados en una búsqueda de los "secretos de la naturaleza". Rara vez mencionan la influencia de factores no genéticos e interacciones múltiples que actúan sobre las causas de las enfermedades, o el cuestionamiento de los objetivos, los métodos o el valor real de la investigación genéti$\mathrm{ca}^{11}$.

Los medios de comunicación y la mercadotecnia de la alimentación no solo ejercen su influencia sobre los individuos, sino también sobre las respuestas que las instituciones públicas dan a los problemas de salud. Y, por supuesto, también sobre los profesionales sanitarios y científicos. Como se ve, el proceso nutrición-alimentación-comunicación-publicidad-consumo es realmente un círculo.

¿Es posible un mundo sin marketing? Probablemente no. $Y$ entonces, ¿por qué no aprovechar sus recursos y técnicas para orientar la opinión y actitudes de la población en la dirección de un mayor interés e implementación de los hábitos de alimentación saludable? ${ }^{1}$. Ciertamente es un objetivo difícil, pero necesario, y en su consecución han de implicarse con responsabilidad políticos, científicos, profesionales e industria. Y los individuos, porque, al final, es la decisión individual de cada uno lo que cuenta (Tabla 1).

En el sentido de la nutrición positiva que se ha mencionado al comienzo de este texto, aparecen, recientemente pero con gran fuerza -estruendo a veces-, los Ilamados alimentos funcionales (aquellos alimentos naturales o elaborados que forman parte de una dieta estándar y a los que se les atribuyen efectos beneficiosos sobre alguna función del organismo o que se afirma reducen el riesgo de enfermar ${ }^{1}$ ). La regulación de estos productos y su publicidad constituye una de las preocupaciones nucleares de las agencias institucionales como la European Food Safety Authority (EFSA) en Europa y la Agencia Española de Seguridad Alimentaria (AESAN) en España. Las declaraciones nutricionales (alegaciones que indican que un producto alimenticio contiene "un nutriente en especial") y las declaraciones saludables (alegaciones de un beneficio específico para la salud por su contenido en cierto nutriente, o bien porque ha demostrado afectar de forma beneficiosa a la salud) son, como muestran E. Baladia, J. Basulto y M. Manera en esta misma Revista ${ }^{12}$, la clave para situar estos productos en el mercado nutricional y promover su consumo. Esta modalidad de mercadotecnia nutricional es de difícil comprensión para el consumidor medio, ya que requiere cierto conocimiento científico, por lo que es terreno abonado para los fraudes y engaños ${ }^{13,14}$. La regulación de estos aspectos, necesaria para la seguridad de consumidores y de las propias industrias responsables, no está libre de vicisitudes y dificultades, sobre todo en la aplicación de la normativa general a las particularidades de la alimentación infantil (y la protección y promoción de la lactancia materna en lugar principal) ${ }^{12}$.

Parece demostrado que la publicidad influye en los hábitos de consumo de la población, y los niños no son, como cabía esperar, una excepción. Es más, probablemente son un grupo de población particularmente vulnerable a los efectos de la publicidad. Los datos actuales parecen mostrar que la publicidad hace que los niños pidan y consuman más productos menos saludables (más ricos en calorías, grasas, azúcares y sal). Aunque no se ha demostrado una relación directa entre la publicidad de este tipo de productos y, por ejemplo, la obesidad, es bastante probable que, al menos, sea un factor relevante ${ }^{15}$. 
Tabla 1. Recomendaciones de alimentación para la prevención de enfermedades crónicas

\section{A nivel individual}

- Mantener un peso saludable:

- Comer cantidades moderadas, adaptadas a las necesidades individuales

- Elegir alimentos ricos en fibra

- Evitar bebidas azucaradas

- Evitar alimentos procesados altamente energéticos y pobres en nutrientes

- Adoptar un estilo de vida físicamente activo:

- Incorporar la actividad física en las rutinas cotidianas

- Participar regularmente en actividades deportivas o de ocio activo

- Limitar las actividades sedentarias

- Realizar actividades físicas de intensidad moderada o elevada varias veces a la semana

- Adoptar hábitos alimenticios saludables:

- Dieta mediterránea

- Al menos cinco raciones de fruta y verduras al día.

- Preferir alimentos con cereales integrales a los refinados

- Tomar legumbres varias veces a la semana

- Usar aceite de oliva, en crudo o para cocinar

- Consumir frutos secos con frecuencia

- Para beber: agua

- Alcohol: poco, y mejor nada

- Evitar: productos con alta densidad energética, con elevado contenido en azúcares y sal; productos con grasas hidrogenadas (ácidos grasos trans); carnes rojas y procesadas; productos ahumados; cocinado a altas temperaturas y con métodos que favorezcan el carbonizado de los alimentos.

\section{A nivel colectivo}

- Información fiable y accesible:

- Etiquetado informativo y comprensible

- Campañas poblacionales ambiciosas y mantenidas

- Formación escolar sobre alimentación

- Control y supervisión sobre la publicidad de los alimentos

- Entornos que faciliten las opciones saludables:

- Producción y distribución de productos saludables a precios asequibles

- Prohibir la publicidad de productos no saludables dirigida a niños

- Menús escolares equilibrados

- Prohibir las máquinas expendedoras de "alimentos" y bebidas en los colegios

- Eliminar las grasas trans de la cadena alimentaria

- Reducir el contenido de azúcar y sal en los productos procesados

Tomado y adaptado de M. A. Royo Bordonada, $2013^{1}$.

Según el posicionamiento de los dietistas canadienses, existe suficiente evidencia para apoyar la necesidad de un abordaje integral y multisectorial para reducir los efectos negativos de la publicidad de alimentos y bebidas "poco saludables" sobre los niños. Para ello, recomiendan: a) que la autorregulación de las empresas de alimentación no es suficiente para reducir el impacto negativo de la publicidad en los niños; b) que deberían establecerse unos estándares basados en criterios científicos para determinar los alimentos "saludables" y los "menos saludables", y este trabajo debería regularse legislativamente; c) que ya que la publicidad televisiva no es la única que ven los niños, las res- tricciones deberían hacerse extensivas a todos los soportes publicitarios existentes, y d) que deberían promocionarse los anuncios de alimentos y bebidas saludables, pues algunas investigaciones apuntan a que esto podría tener efectos positivos sobre la preferencia hacia estos productos ${ }^{16}$.

En la situación que se ha descrito, se hace evidente la necesidad de nuevas formas y fuentes de información apoyadas en las tecnologías de la información y la comunicación (TIC) y los presupuestos de la Web 2.0. Y aquí es justo y obligado citar que no pocos blogs desarrollados con rigor por entusiastas científicos y profesionales de la nutrición y la 
dietética tratan de poner una ración de ciencia y racionalidad en esta intrincada selva de la alimentación y la publicidad. Ocupan un lugar que no saben o no pueden hacer las publicaciones científicas clásicas, como esta Revista. Aquí citamos unos pocos $^{17,18}$, pero sabiendo que es solo una muestra y que no están todos los que son; animamos a los lectores a que busquen otros, interactúen y cola-

\section{BIBLIOGRAFÍA}

1. Royo Bordonada MA (ed). La alimentación y el consumidor. Madrid: Escuela Nacional de Sanidad, Instituto de Salud Carlos III, Ministerio de Economía y Competitividad; 2013 [en línea] [consultado el 11/11/ 2013]. Disponible en http://goo.gl/mXIHOn

2. Revuelta G. Salud y medios de comunicación en España. Gac Sanit. 2006;20(Supl 1):203-8.

3. YMEDIA. Medios de comunicación, España, enero de 2013 [en línea] [consultado el 13/11/2013]. Disponible en www.ymedia.es/es/mapa-de-medios

4. Amador Romero FJ. Medios de comunicación y opinión pública sanitaria. Aten Primaria. 2004;33(2):95-8.

5. Montes de Oca A. La comunicación social en la promoción de la salud. Rev Esp Nutr Comunitaria. 2010;16:9-12

6. Basulto J, Caorsi L. Consejos nutricionales en los diarios, ¿son fiables? Eroski Consumer, 1 de octubre de 2013. [en línea] [consultado el 13/11/2013]. Disponible en http://goo.gl/JU8LKd

7. Un vaso de vino al día reduce el riesgo a tener una depresión. Europapress.es Salud, 2 de septiembre de 2013 [en línea] [consultado el 13/11/2013]. Disponible en http://goo.gl/X7UmUJ

8. Gea A, Beunza JJ, Estruch R, Sánchez-Villegas A, SalasSalvadó J, Buil-Cosiales P, et al. Alcohol intake, wine consumption and the development of depression: the PREDIMED study. BMC Med. 2013;11:192 [en línea]. Disponible en www.ncbi.nlm.nih.gov/pmc/ar ticles/PMC3765610/

9. Basulto J. ¿Vino para la depresión? Madre mía. SER Consumidor, 12 de septiembre de 2013 [en línea] [consultado el 13/11/2013]. Disponible en http:// goo.gl/o2Pd6W

10. Cooper BE, Lee WE, Goldacre BM, Sanders TA. The quality of the evidence for dietary advice given in UK boren en la difusión de los mismos entre sus círculos profesionales. Como ocurre con estos blogs, los profesionales sanitarios necesitamos reforzar el escepticismo, la racionalidad, la modestia, el sentido común (el propio y la confianza en el ajeno) y la saludable impertinencia científica ${ }^{19,20}$ a la hora de abordar la alimentación infantil.

national newspapers. Public Underst Sci. 2012;21(6): 664-73.

11. Petersen A. Biofantasies: genetics and medicine in the print news media. Soc Sci Med. 2001;52(8):1255-68.

12. Baladia E, Basulto J, Manera M. Declaraciones nutricionales y de propiedades saludables aplicables a los productos de alimentación infantil en España y en la Unión Europea. Rev Pediatr Aten Primaria. 2013;15: 351-9.

13. Alimentación infantil: cuidado con los bulos y la publicidad engañosa. Wikilibros. [en línea] [consultado el 28/11/2013]. Disponible en http://goo.gl/Cvtyuv

14. Neo Peques, el herbolario para el niño y la niña. El blog de Centinel, 11 de noviembre de 2013 [en línea] [consultado el 13/11/2013]. Disponible en http:// goo.gl/EFvCeU

15. Basulto J. Se me hace bola. Barcelona: Ed. Debolsillo; 2013.

16. Dietitians of Canada. Advertising of Food and Beverages to Children. Position of Dietitians of Canada, 2010 [en línea] [consultado el 20/11/2013]. Disponible en http://goo.gl/ielrUK

17. Revenga J. El nutricionista de la general [en línea] [consultado el 13/11/2013]. Disponible en http:// blogs.20minutos.es/el-nutricionista-de-la-general/

18. Basulto J, Carpio A, Ortí A. Comer o no comer [en línea] [consultado el 13/11/2013]. Disponible en http://comeronocomer.es/

19. Schwarz MJ. Quizá este blog no es para usted. El retorno de los charlatanes, 03/09/2009 [en línea] [consultado el 28/11/2013]. Disponible en http://charlatanes.blogspot.com.es/2009/09/quiza-este-blog -no-es-para-usted.html

20. López Nicolás JM. SCIENTIA [en línea] [consultado el 28/11/2013]. Disponible en http://scientiablog. com/ 